\title{
Antimicrobial Activity of Probiotics on Swiss Mice
}

\author{
Najett Menad ${ }^{1}$, Ahmed Bensoltane ${ }^{1}$, Akil Loumani ${ }^{1}$, Djamila Maghnia ${ }^{1}$, Nachida Slimane ${ }^{1}$, Fadela Chougrani $^{2}$ \\ and Abderrahim Cheriguene ${ }^{2}$ \\ 1. Laboratory of Food and Industrial Microbiology, Department of Biology, Faculty of Science, University of Oran, Es-Senia Oran \\ 31000, Algeria \\ 2. Laboratory of Microbiology, Department of Biology, Faculty of Natural Sciences and Life, University of Mostaganem, B.P. 227 \\ Mostaganem 27000, Algeria
}

Received: August 11, 2011 / Published: July 20, 2012.

\begin{abstract}
Salmonella enterica serovar typhimurium is the first cause identified of food poisoning in humans; new data indicate that the resistant strains of Salmonella sp. can be inhibited by a bacterial population present in foods have been called probiotics. Our study was conducted on Swiss albino mice in order to know the effect of probiotics or biotherapeutic agents, and suggest the different therapeutic possibilities of intestinal complications related to the presence of Salmonella enterica serovar typhimurium; Reference strains, Lactobacillus delbrueckii subsp. bulgaricus, Lactobacillus acidophilus and Lactobacillus paracasei in combination with Bifidobacterium sp. were tested for their health benefits. The number of Salmonella enterica serovar typhimurium decreases in intestine, in the stomach and in the liver for group 2, 3 in case of preventive treatment and group 4, 5 in case of a therapeutic treatment due to the use of a culture of probiotics, in comparison with group 1 where we recorded a high number. The bio-therapeutic treatment was finally confirmed by macroscopic and microscopic observations of different target organs before and after treatment for the experiment period.
\end{abstract}

Key words: Bifidobacterium sp., Lactobacillus sp., Salmonella enterica serovar typhimurium, probiotics, antagonism in vivo.

\section{Introduction}

An imbalance of intestinal flora may be one expression of intestinal disorders (diarrhea) and infections caused by pathogenic bacterial agents.

These diseases have highlighted the crucial role of intestinal microflora in health improvement. This microflora is strongly influenced by diet [1].

Indeed, food is likely to modulate various functions of the body, and may also help to reduce the risk of certain diseases. In recent years, many studies have been undertaken to assess the health impact of certain dietary components to non-nutrition such as probiotics [2].

Probiotics used are live strains, such as Lactobacilli. These lactobacilli have the capacity to produce substances that are impressive to have an antagonistic

Corresponding author: Najett Menad, researcher, research field: food microbiology. E-mail: na_11011986@yahoo.fr, msn-nadjet-1@hotmail.fr. activity against other pathogenic bacterial species [1] Apart from lactobacilli, there is a new species of probiotics; it is Bifidobacteria.

Our study was performed on some bacteria used as probiotics, in order to control their beneficial effects, and it is necessary to achieve these studies into animal to understand their effects [1].

In this work, we propose to test in vivo the colonization, establishment in the gut, translocation and the antagonistic effect to reference strains belonging to the genus Lactobacillus sp. in combination with a strain Bifidobacterium sp. against Salmonella enterica serovar typhimurium, this was performed by measuring the viability of the latter.

\section{Materials and Methods}

\subsection{Materials}

Three reference strains were used for this study: Lactobacillus delbrueckii subsp. bulgaricus (CNRZ 
208T), Lactobacillus acidophilus (LMG 11430), Lactobacillus paracasei (ATCC 334T), were provided by professor Françoise Berthier Researcher at the INRA station Poligny (France). They were revived and subcultured in MRS broth-lactose at $\mathrm{pH}$ 5.5, and then isolated on the same medium solid (2\% agar) [3].

Bifidobacterium sp. strain has been isolated by us from samples of yoghurt (Danone bioactevia, Algeria) on Columbia medium agar modified [3].

Regarding the pathogenic strain Salmonella enterica serovar typhimurium, it was provided by the laboratory: group poultry west «ORAVIO» Mostaganem (Algeria).

Mice used in this protocol were albino mice strain NMRI Swiss aged from 6 to 9 weeks and provided by Pasteur Institute of Algeria.

Water, was sterilized by autoclaving and used as culture medium for monitoring the in vivo antagonism.

\subsection{Methods}

Authors have planned 6 groups of 7 mice (Fig. 1).

The first part is the study of initial digestive flora.

The second part deals with infected mice that are subjected to different treatments to track the implementation of probiotics in organs and their contents and control their effects against Salmonella enterica serovar typhimurium (was isolated on XLD agar).

The mice in each group were subjected to a sacrifice that collects organs infected by the following steps: fixation in BOUIN, dehydration, clarification and impregnation in paraffin, inclusion, preparation cuts, coloring, editing (Hematoxylin-Eosin).

\section{Results}

According to bacteriological analysis of various organs and their contents of 6 groups before any experiment (day 0) revealed no presence of Salmonella enterica serovar typhimurium, but authors noted the presence of the lactic flora for each group (Fig. 2).

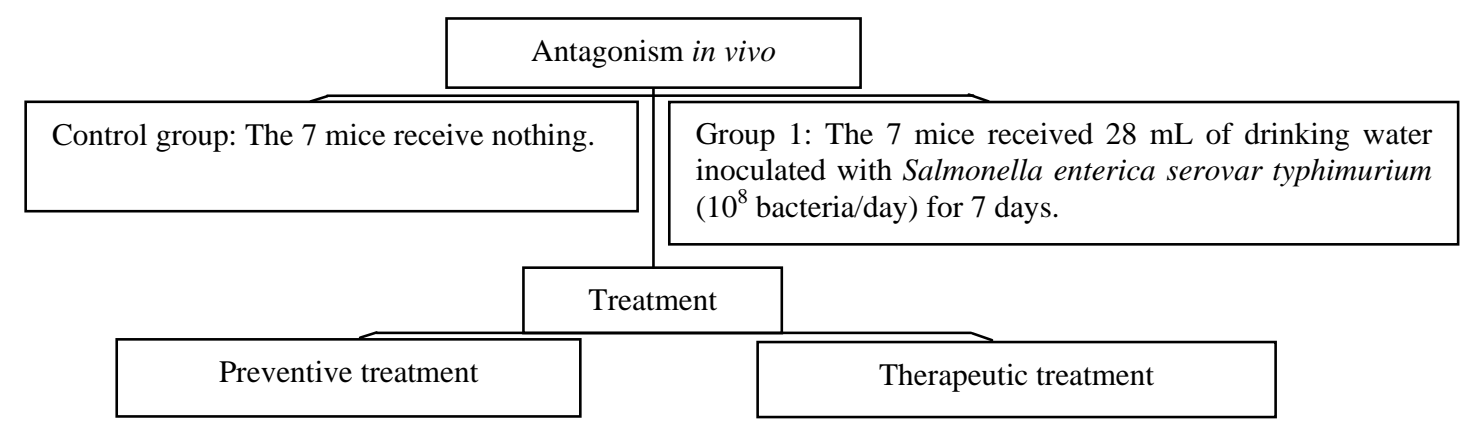

Group 2: The 7 mice were administered $28 \mathrm{~mL}$ of drinking water inoculated with Bifidobacterium sp. $\left(10^{8}\right.$ bacteria/day) in mixed culture with Lactobacillus delbrueckii subsp. bulgaricus $\left(10^{8}\right.$ bacteria/day) + Lactobacillus acidophilus $\left(10^{8}\right.$ bacteria/day $)+$ Lactobacillus paracasei $\left(10^{8}\right.$ bacteria/day) for a week; then they are given $28 \mathrm{~mL}$ of drinking water inoculated with Salmonella enterica serovar typhimurium during the second week.

Group 3: The 7 mice were administered $28 \mathrm{~mL}$ of drinking water with three strains of Lactobacillus sp. only: Lactobacillus delbrueckii subsp. bulgaricus $\left(10^{8}\right.$ bacteria/day) + Lactobacillus acidophilus $\left(10^{8}\right.$ bacteria/day) + Lactobacillus paracasei $\left(10^{8}\right.$ bacteria/day) for a week then $28 \mathrm{~mL}$ of drinking water inoculated with Salmonella enterica serovar typhimurium during the second week.
Group 4: The 7 mice received $28 \mathrm{~mL}$ of drinking water inoculated with Salmonella enterica serovar typhimurium ( $10^{8}$ bacteria/day) for 48 hours. Then $28 \mathrm{~mL}$ of drinking water cultured with Bifidobacterium sp. $\left(10^{8}\right.$ bacteria/day) in mixed culture with Lactobacillus delbrueckii subsp. bulgaricus $\left(10^{8}\right.$ bacteria/day) + Lactobacillus acidophilus $\left(10^{8}\right.$ bacteria/day $)+$ Lactobacillus paracasei $\left(10^{8}\right.$ bacteria/day) for a week.

Group 5: The 7 mice were administered $28 \mathrm{~mL}$ of drinking water inoculated with Salmonella enterica serovar typhimurium $\left(10^{8}\right.$ bacteria/day) for 48 hours. Then $28 \mathrm{~mL}$ of drinking water cultured with the three strains of Lactobacillus sp. alone: Lactobacillus delbrueckii subsp. bulgaricus $\left(10^{8}\right.$ bacteria/day $)+$ Lactobacillus acidophilus $\left(10^{8}\right.$ bacteria/day $)+$ Lactobacillus paracasei $\left(10^{8}\right.$ bacteria/day) for a week.

Fig. 1 In vivo antagonism of 3 species: Lactobacillus delbrueckii subsp. bulgaricus, Lactobacillus acidophilus, Lactobacillus paracasei alone or in combination with Bifidobacterium sp. against Salmonella enterica serovar typhimurium in a preventive and therapeutic treatment. 
In these organs, there was an increase in the number of germs in stomach $\left(0.442 \times 10^{6} \mathrm{CFU} / \mathrm{g}, 0.049 \times 10^{6}\right.$ $\mathrm{CFU} / \mathrm{g}, 0.775 \times 10^{6} \mathrm{CFU} / \mathrm{g}$ and $0.157 \times 10^{6} \mathrm{CFU} / \mathrm{g}$ ) for groups 2, 3, 4 and 5 respectively and in intestine and its contents $\left(0.419 \times 10^{6} \mathrm{CFU} / \mathrm{g}, 0.047 \times 10^{6} \mathrm{CFU} / \mathrm{g}\right.$, $0.562 \times 10^{6} \mathrm{CFU} / \mathrm{g}, 0.260 \times 10^{6} \mathrm{CFU} / \mathrm{g}$ ) for groups 2,3 , 4 , and 5 respectively and in colon and its contents, an increase in number of $1.237 \times 10^{6} \mathrm{CFU} / \mathrm{g}, 0.045 \times 10^{6}$ CFU/g, $2.337 \times 10^{6} \mathrm{CFU} / \mathrm{g}$ and $0.110 \times 10^{6} \mathrm{CFU} / \mathrm{g}$ compared with control group $\left(0.007 \times 10^{6} \mathrm{CFU} / \mathrm{g}\right.$, $0.002 \times 10^{6} \mathrm{CFU} / \mathrm{g}, 0.038 \times 10^{6} \mathrm{CFU} / \mathrm{g}$ ) (Fig. 2a).

From Fig. 2a, our observations indicate that probiotics used were found in the stomach, intestine and colon with high degree of implantation compared to control group, which indicates the reduction of the pathogen in the organs and their contents of group 2 $\left(0.015 \times 10^{4} \mathrm{CFU} / \mathrm{g}, 0.038 \times 10^{4} \mathrm{CFU} / \mathrm{g}, 0.075 \times 10^{4}\right.$ $\mathrm{CFU} / \mathrm{g}), 4\left(0.078 \times 10^{4} \mathrm{CFU} / \mathrm{g}, 0.007 \times 10^{4} \mathrm{CFU} / \mathrm{g}\right.$, $0.353 \times 10^{4} \mathrm{CFU} / \mathrm{g}$ ) which received the drinking water inoculated with Bifidobacterium sp. in mixed culture with three strains of Lactobacillus sp. (Fig. 2b), for group $3\left(0.104 \times 10^{4} \mathrm{CFU} / \mathrm{g}, 0.228 \times 10^{4} \mathrm{CFU} / \mathrm{g}, 0.445\right.$ $\left.\times 10^{4} \mathrm{CFU} / \mathrm{g}\right)$ and $5\left(0 \mathrm{CFU} / \mathrm{g}, 0.083 \times 10^{4} \mathrm{CFU} / \mathrm{g}\right.$, $0.227 \times 10^{4} \mathrm{CFU} / \mathrm{g}$ ) which received only three Lactobacillus sp. (Fig. 2b) when compared with results of dissection, mice of group 1 that received Salmonella enterica serovar typhimurium alone $\left(0.104 \times 10^{4} \mathrm{CFU} / \mathrm{g}\right.$, $0.228 \times 10^{4} \mathrm{CFU} / \mathrm{g}, 0.445 \times 10^{4} \mathrm{CFU} / \mathrm{g}$ ) (Fig. 2b).

This pathogenic species is absent in all organs of the control group (Fig. 2b).

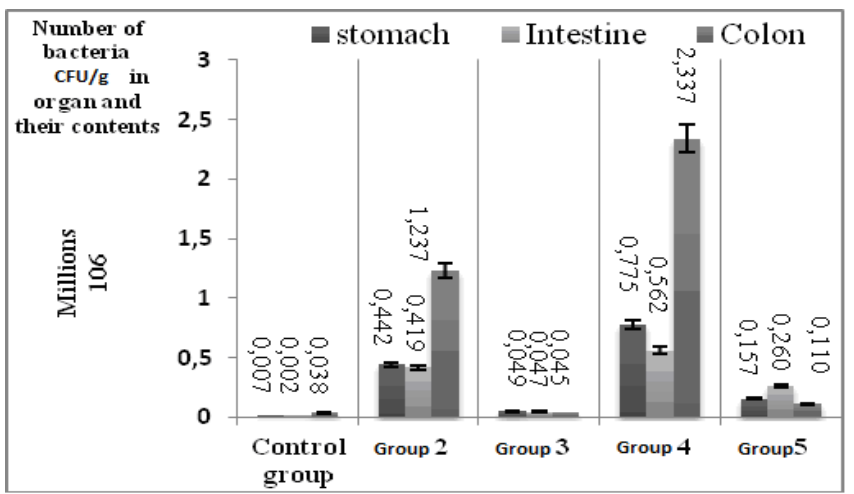

(a)

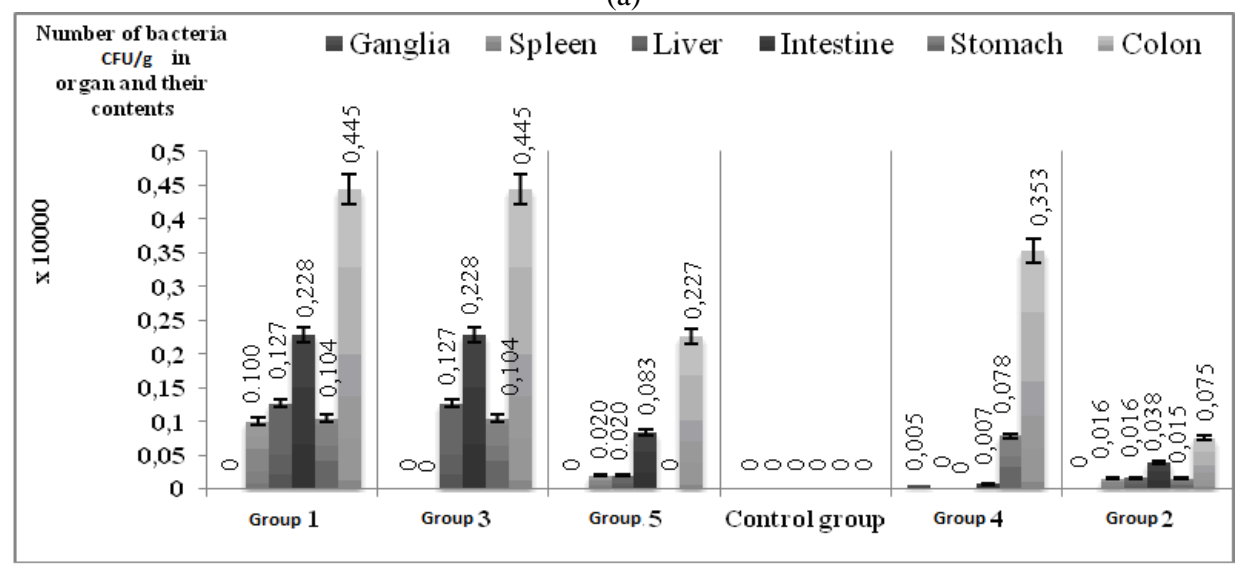

(b)

Fig. 2 (a) Degree of implementation of probiotic strains in the digestive organs and their contents of mice sacrificed during and after 14 days. (b) Rates of Salmonella enterica serovar typhimurium in all organs and their contents of 6 groups for 14 days.

The results of histopathological analysis in the intestine of mice in group 1, authors detected an enteritis with the presence of neutrophils, and at the villus was observed as a cluster bacillary reddish (Fig. 3a, group 1). If group 2 is observed the neutrophils as we noted a mobilization of lymphocytes (Fig. 3a, 
group 2). For group 3, it is recorded a secretion and mobilization of lymphocytes in the lamina propria. Authors also noted an immunostimulatory effect resulting in swelling of the plates paying through the germination of immune cells (Fig. 3a, group 3 ). In the intestine of mice in group 4 (Fig. 3a, group 4) the tissue remains as it is with an architecture identical to the gut in control group (Fig. 3a, control group), for group 5, it is recorded the presence of a reddish mass bacillary (Fig. 3a, group 5).

Microscopic observation of liver cross section in group 1 indicates that there is liver damage with presence of neutrophils (Fig. 3b, group 1). In the liver of a mouse in group 2, the tissue remains normal and there is presence of neutrophils but fewer in number (Fig. 3b, group 2) relation to group 3, where the number is more important at the sinusoids (Fig. 3b, group 3). In the liver of a mice in group 4, a total absence of the pathogen, and the tissue remains normal (Fig. 3b, group 4) and identical to control group (Fig. $3 b$, control group) at group 5 , it is recorded the presence of neutrophils (Fig. 3b, group 5).

Microscopic study as cross sections of the spleen, showed the presence of splenitis with neutrophils in the spleen of mice in group 1 (Fig. 3c, group 1). For the spleen of a mice in group 3 and group 5 was a strong presence of neutrophils (Fig. 3c, group 3 and group 5) when compared with group 2, there was a reduction of the lymphocyte population with immune stimulation (Fig. 3c, group 2) and at the group 4 was observed that there is an absence of immune cell (Fig. 3c, group 4) with a tissue architecture similar to control group (Fig. 3c, control group).

\section{Discussion}

After ingestion of probiotics chosen for the duration of experimentation authors observed an increase of the lactic flora in the organs and their contents (Fig. 2a).

Administered lactobacilli are able to quantitatively modify the intestinal microflora in animals during withdrawal and this is probably due to the survivability of Lactobacillus acidophilus in acidic conditions which

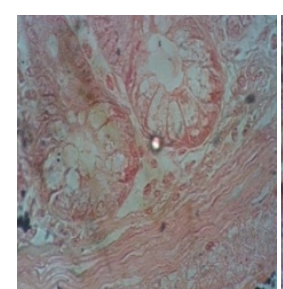

(a) Control group

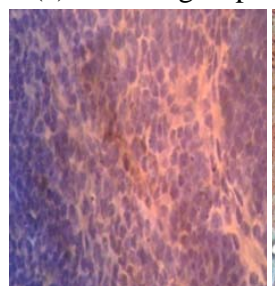

(b) Control group

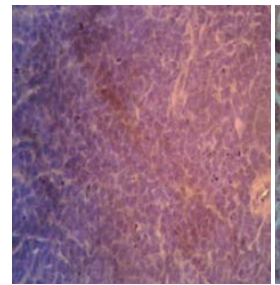

(c) Control group

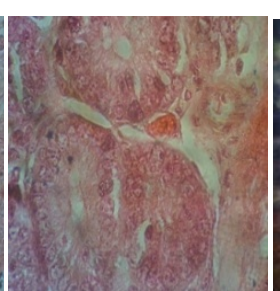

Group 1

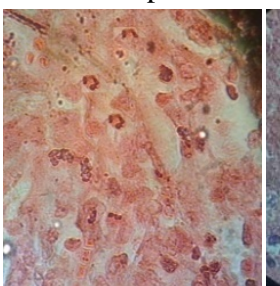

Group 1

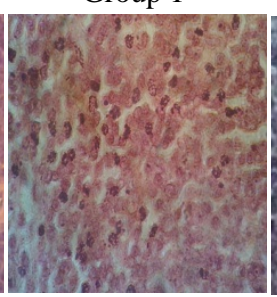

Group 1

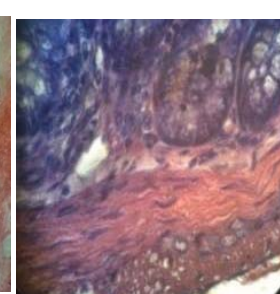

Group 2

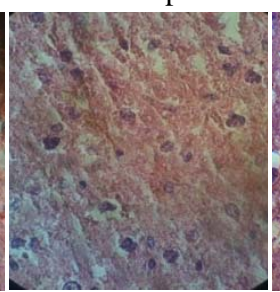

Group 2

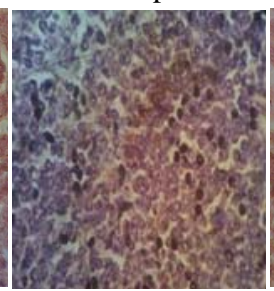

Group 2

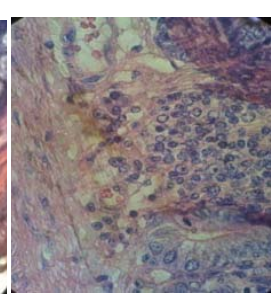

Group 3

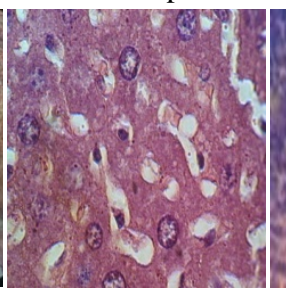

Group 3

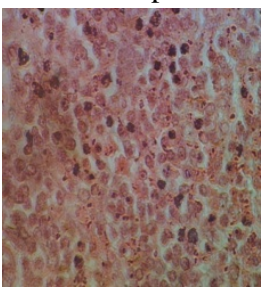

Group 3

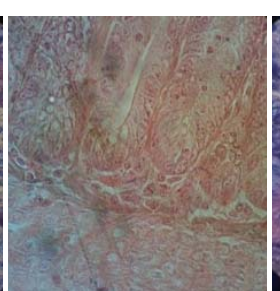

Group 4

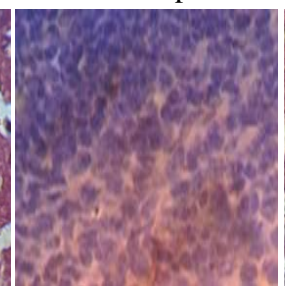

Group 4

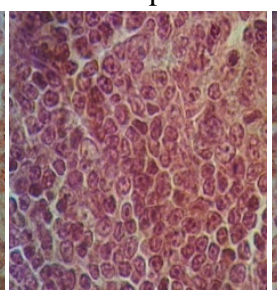

Group 4

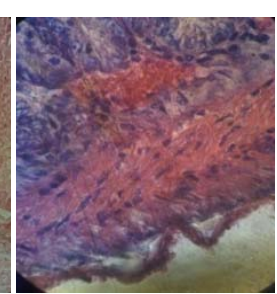

Group 5

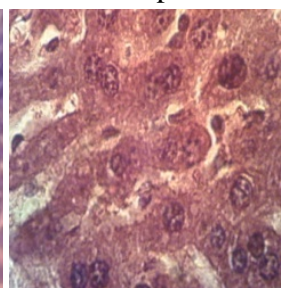

Group 5

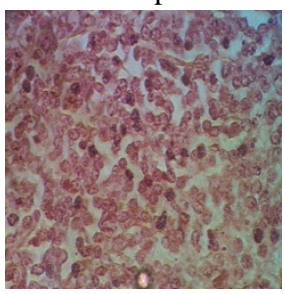

Group 5

Fig. 3 (a) A cross section of a mouse intestine in each group ( $\times$ 100), (b) A cross section of a mouse liver in each group ( $\times$ 100), (c) A cross section of a mouse spleen in each group $(\times 100)$. 
are generally better [4] than strains of Lactobacillus delbrueckii subsp. bulgaricus with low resistance to acid and are therefore rapidly destroyed in the stomach.

The intestine is slightly colonized in our case, the conditions of $\mathrm{pH}$ changes are unfavorable, as well as transit.

The bacterial population increases. pancreatic electrolyte secretion is rich in bicarbonates, it can buffer acidity from the stomach and thus to obtain an optimal $\mathrm{pH}$ is probably for this reason that our probiotics strains are present. And even more in the colon and its contents, which is a place of intense fermentation causing qualitative and quantitative changes of the colonic flora.

The study of animal models has demonstrated that certain probiotic bacteria could inhibit the growth of pathogenic bacteria such as Salmonella enterica serovar typhimurium [5].

Indeed, the results indicate a decrease in number of pathogenic bacteria in case of combination of four different strains relation to group 1 (Fig. 2b).

It is also extremely important to note that all strains of lactic acid bacteria do not possess all the properties [6] for this reason the intake of a probiotic mixture induces a reduction of pathogenic bacteria number; this was found in the results of groups 2 and 4 compared with 3 and 5 (Fig. 2b). Authors observed that administration of Lactobacillus sp. and Bifidobacterium sp. is likely to inhibit the adhesion of Salmonella enterica serovar typhimurium [7].

Among the probiotics strains Lactobacillus acidophilus, can produce their bacteriocins. These bactériocinogènes [8] could also suppress the growth of pathogenic gastrointestinal bacteria, Salmonella sp..

The decrease in the number of Salmonella enterica serovar typhimurium can be achieved by several mechanisms, among which may be given in particular the modulation of the immune system and stimulation of chemotaxis of macrophages [9].

Stimulation of the immune response leads to secretion of secretory IgA.
Similarly, Lactobacillus delbrueckii subsp. bulgaricus stimulates the phagocytic function of macrophages in vivo by the production of an extracellular neutral phosphopolysaccharide [10].

Treatment with a suspension of probiotic strains increases the rate of anti-Salmonella immunoglobulin $A$ and decreases the number of Salmonella in the liver and spleen [11].

In the intestine of group 1 we observed a cluster as bacillary reddish probably due to the presence of the pathogen, when multiplied, resulting in an inflammatory reaction in neutrophils stimulated by chemokines [12], released by intestinal cells.

In the liver, the observation made cuts shows an aspect of the liver of toxic hepatitis which is likely due to the translocation of the pathogen that represents a model of invasive enteric infection, and the spleen was a splenitis.

The mobilization of cells for secretion of IgA may be due to recognition of the microflora of the host immune system resulting in effect through the production of local and systemic antibody [13]. Comparing with the group 3, the number and nature of probiotics administered did not inhibit the establishment and growth of the pathogen in the intestine, which indicates its presence in the liver, spleen.

Our results obtained the spleen of each group $(2,3,5)$ there is a presence of neutrophils which is an indicator that there's a translocation of Salmonella enterica serovar typhimurium in the presence of treatment with different strains of Lactobacillus sp., stimulating the phagocytic capacity of host macrophages [14].

\section{Conclusions}

It was concluded that probiotics or biotherapeutic agents for the treatment of certain microbial infections in humans and certain animal populations improve health and ensure the maintenance of ecological balance of gut microflora, such interest benefits were clearly observed in this study.

It was concluded that an exogenous probiotic 
microorganism alters the composition of the intestinal flora. The results clearly indicate that the probiotic strains tested implanted high level in different organs of the digestive tract, and it was concluded that the speed of this implementation is a criterion for selection of probiotic strains.

The highest rate of implementation of our probiotic strains in fact reflect the protective action developed by the strains tested against Salmonella enterica serovar typhimurium and show that colonization of the gut by these strains of mice can reduce the number of the pathogenic bacteria that was used and change the course of infection showed that the effectiveness of probiotic therapy lies in the ability of growth and implementation of himself.

The existence of a strong antagonist activity of probiotic strains tested, this confirms the presence of one of the most important criteria in selecting probiotic strains, which paves the way for the introduction of these strains in the human diet to prevent diseases.

\section{References}

[1] H.C. Lin, B.H. Su, A.C. Chen, T.W. Lin, C.H. Tsai, T.F. Yeh, et al., Oral probiotics reduce the incidence and severity of necrotizing enterocolitis in very low birthweight infants, Pediatrics 115 (2005) 1-4.

[2] A. Ait-Belgnaoui, W. Han, F. Lamine, H. Eutamene, J. Fioramonti, L. Bueno, et al., Lactobacillus farciminis treatment suppresses stress-induced visceral hypersensitivity: A possible action through an interaction with epithelial cells cytoskeleton contraction in rats, Gut 55 (8) (2006) 1990-1994.

[3] J.P. Larpent, M. Larpent-Gourgaud, Memento technique de microbiologie, chapitre VII: Les microorganismes utiles, Lavoisier Tec \& Doc, Paris, 1997, pp. 753-877.

[4] P. Marteau, F. Shanahan, Basic aspects and pharmacology of probiotics: An overview of pharmacokinetics, mechanisms of action and side-effects, Bailliere's Best Practice and Research in Clinical Gastroenterology 17 (2003) 725-740.

[5] S. Hudault, V. Lievin, M.F. Bernetcamamrd, A.L. Servin,
Antagonistic activity exerted in vivo by Lactobacillus casei (GG) against Salmonella typhimrium C5 infection, Appl. Environ. Microbial 63 (1997) 513-518.

[6] L. De Vuyst, L. Avonts, E. Makras, Probiotics, prebiotics and gut health, in: C. Remacle, B. Reusens (Eds.), Functional Foods: Ageing and Degenerative Disease, Woodhead Publishing Ltd., Cambridge, United Kingdom, 2004, pp. 416-482.

[7] G. Chauvière, M.H. Coconnier, S. Kernéis, J. Fourniat, A.L. Servin, Adhesion of human Lactobacillus acidophilus strain LB to human enterocyte-like Caco-2 cells, J. Gen. Microbiol. 138 (1992) 1689-1696.

[8] M.H. Cocconier, V. Lievin, E. Hemery, A.L. Servin, Antagonistic activity against Helicobacter infection in vitro and in vivo by the human Lactobacillus acidophilus strain, L.B. Appl. and Environ. Microbial 64 (11) (1998) 4573-4580.

[9] H. Kitazawa, T. Ino, Y. Kawai, T. Itoh, T.A. Saito, Novel immunostimulating aspect of Lactobacillus gasseri: Induction of «Gasserokine» as chemoattractants for macrophages, Int. J. Food Microbiol. 77 (1-2) (2002) 29-38.

[10] H. Kitazawa, Y. Ishii, J. Uemura, Y. Kawai, T. Saito, T. Kaneko, et al., Augmentation of macrophage functions by an extracellular phosphopolysaccharide from Lactobacillus delbrueckii ssp., Bulgaricus, Food Microbiology 17 (2000) 109-118.

[11] G. Perdignon, M.E. Nader, M.C. Apella, S. Alvarez, G. Oliver, A.A. Pesce de Ruis Holgadon, Prevention of gastrointestinal infection using immunobiological methods with milk fermented by Lactobacillus casei and Lactobacillus acidophilus, J. Dairy Res. 57 (1992) 255264.

[12] B.A. Mc Cormick, Transepithelial signaling to neutrophils by Salmonellae: A novel virulence mechanism for gastroenteritis, Infect Immun. 63 (1995) 2302-2309.

[13] H.Z. Apperloo-Renkema, T.G. Jagt, R.H. Tonk, D. Van Der Waaij, Healthy individuals possess circulating antibodies against their indigenous faecal microflora as well as against allogenous faecal microflora: An immunomorphometrical study, Epidemiol. Infect 111 (1993) 273-85.

[14] E. Neumann, M.A.P. Oliveira, C.M. Cabral, L.N. Moura, J.R. Nicoli, C.E. Vieira, et al., Monoassociation H2B20 with Lactobacillus acidophilus stimulates the immune defense mechanisms of germfree mice, Brazilian Journal of Médical Research and Biological 31 (1998) 1565-1573. 